\title{
RUBBING FASTNESS OF GREEN INK PRINTED ON TEXTILE USING SCREEN PRINTING TRANSFER TECHNIQUE
}

\author{
Ana Lilić (D), Nemanja Kašiković (iD, Nada Miketić (DD \\ University of Novi Sad, Faculty of Technical Sciences, \\ Department of Graphic Engineering and Design, Novi Sad, Serbia
}

\begin{abstract}
Printing on textile surfaces is possible using either direct or transfer method. The transfer method is a complex technique in which, the first, design is transferred to a flexible substrate via digital or screen printing, after which the transfer to textiles is carried out. To apply the colour to the material, heat pressure is used. Different environmental influences can affect such printed material. Some of them are rubbing, heat, UV radiation, moisture, washing, etc. In this study, an analysis of the impact that rubbing has on the print will be done. Rubbing treatment of the prints was done according to ISO 105-X12 standard. Colour changes of the printed textile materials to dry rubbing treatment were characterized by the spectrophotometric measurements. In the given analysis, two different types of cotton material were used as a printing medium. Both materials are $100 \%$ cotton, however, they differ in the weight of the fabric. The first material has a weight of $150 \mathrm{~g} / \mathrm{m}^{2}$ and the other has $190 \mathrm{~g} / \mathrm{m}^{2}$.
\end{abstract}

Key words: rubbing, transfer printing, textile, colour difference

\section{INTRODUCTION}

Textile printing can be best described as the art and science of decorating a fabric with a colourful pattern or design (Tippet, 2005). The saga of digitally printing and dyeing of fabrics, yarns and garments involves a past of a few decades, a dynamic present and likely a bright future. This introduction accounts for the origins and evolution of textile printing to digital solutions. It identifies some of the many creators and pioneers of these technologies and assesses their impact on the textile printing industry (Cahil, 2006). Printing on textile surfaces is possible using either direct or transfer method. Transfer printing is the term used to describe textile and related printing processes in which the design is first printed on to a flexible non-textile substrate and later transferred by a separate process to a textile. Transfer printing process could be divided into: sublimation transfer, melt transfer, film release and wet transfer. The reasons why this technique is still popular are numerous. The production of short runs and repeated orders is much easier to produce, sometimes it is easier to produce complex design on the paper than on the textile, designs could be printed on cheap substrate before the transfer to the more expensive textile materials, and sometimes this is the only way to make certain special effects on garments or garment panels. The designs that are printed on paper, decrease both storage space and costs, as relatively cheap equipment is needed (printing machines, irons, etc.), Later, the design may be applied to the textiles with relatively low skill input and low reject rates (Rattee, 2003). The clothes are usually exposed to external influences such as washing, heat, abrasion, UV light, etc. (Kašiković et al, 2017). The resistance of the material to rubbing will be analysed in this article. Samples will be exposed to the rubbing procedure and afterwards will be measured the quality of the print and compared with the original. As a result, the effect of rubbing will be shown and whether it affects the visual quality of the print.

\section{MATERIALS AND METHODS}

In the given analysis, two different types of cotton material were used as a printing medium. Properties of these materials are shown in Table 1.

An appropriate test map consisting of three fields of size $12 \times 5 \mathrm{~cm}$ was prepared for study. Heat transfer papers $\left(150 \mathrm{~g} / \mathrm{m}^{2}\right)$ were printed using screen printing technique ( $M$ \& R Sportsman E Series, printing speed was $15 \mathrm{~cm} / \mathrm{sec}$, the hardness of the squeegee $80^{\circ}$ Shore Type $A$, the printing pressure $275.8 \times 10^{3} \mathrm{~Pa}$ and the snap-off distance $4 \mathrm{~mm}, 55$ and 90 threads/cm). The ink used for printing the transfer paper is Sericol Texopaque Classic OP Plastisol. Afterwards, from the transfer paper, the test card was transferred to the textile material by heat pressure for 10 seconds. Three different temperatures were used for this process $\left(160,170\right.$ and $\left.180^{\circ} \mathrm{C}\right)$. 
Electronic crockmeter Testex textile instrument LTD. TF411 (rubbing head diameter $16 \mathrm{~mm}$, vertical pressure $9 \mathrm{~N}$, rubbing stroke $104 \mathrm{~mm}$, according to the ISO 105x12/D02 standard) was used as an instrument for analysis.

Spectrophotometric measurements and visual control of printed samples were performed after the printing process and after exposure to the rubbing effect, the samples being subjected to rubbing at 500, 1000 and 1500 times. Colorimetric measurements were made using the HP 200 measuring device (D65 lighting, $2^{\circ}$ standard observer, $\mathrm{d} / 8$ measuring geometry).

Colour differences between treated and untreated samples were calculated using $\Delta \mathrm{E} 94$ formula. Colour difference value can be translated to human perception reference as $0<\Delta E<1$ - the difference cannot be noticed; $1<\Delta \mathrm{E}<2$ - small colour difference, visible to "trained" eye; $2<\Delta \mathrm{E}<3,5$ - Medium colour difference, visible to "untrained" eye; $3,5<\Delta E<5$ - Obvious colour difference; $\Delta \mathrm{E}>5$ - Massive colour difference (Novaković, 2008).

Untreated and treated samples were scanned using a Canon CanoScan 5600F scanner that was set to 600 spi resolution without auto correction function.

Table 1: Properties of the used textile materials

\begin{tabular}{|c|c|c|c|c|c|}
\hline Tests & Type of weaves & $\begin{array}{c}\text { Material } \\
\text { composition } \\
\text { (\%) }\end{array}$ & Fabric weight & \multicolumn{2}{|c|}{ Thread count $\left(\mathrm{cm}^{-1}\right)$} \\
\cline { 3 - 6 } & Single & Cotton 100\% & 150 & Vertical & Horizontal \\
\hline Material 1 & Single & Cotton 100\% & 190 & 15 & 19 \\
Material 2 & & & ISO 1833 & ISO 3801 & ISO 7211-2 \\
\hline Method & & & \multicolumn{3}{|r}{} \\
\hline
\end{tabular}

\section{RESULTS AND DISCUSSION}

In Figures 1 and 2 are presented values of the colour difference between the samples immediately after printing and after rubbing procedure which occurred 500, 1000 and 1500 times.

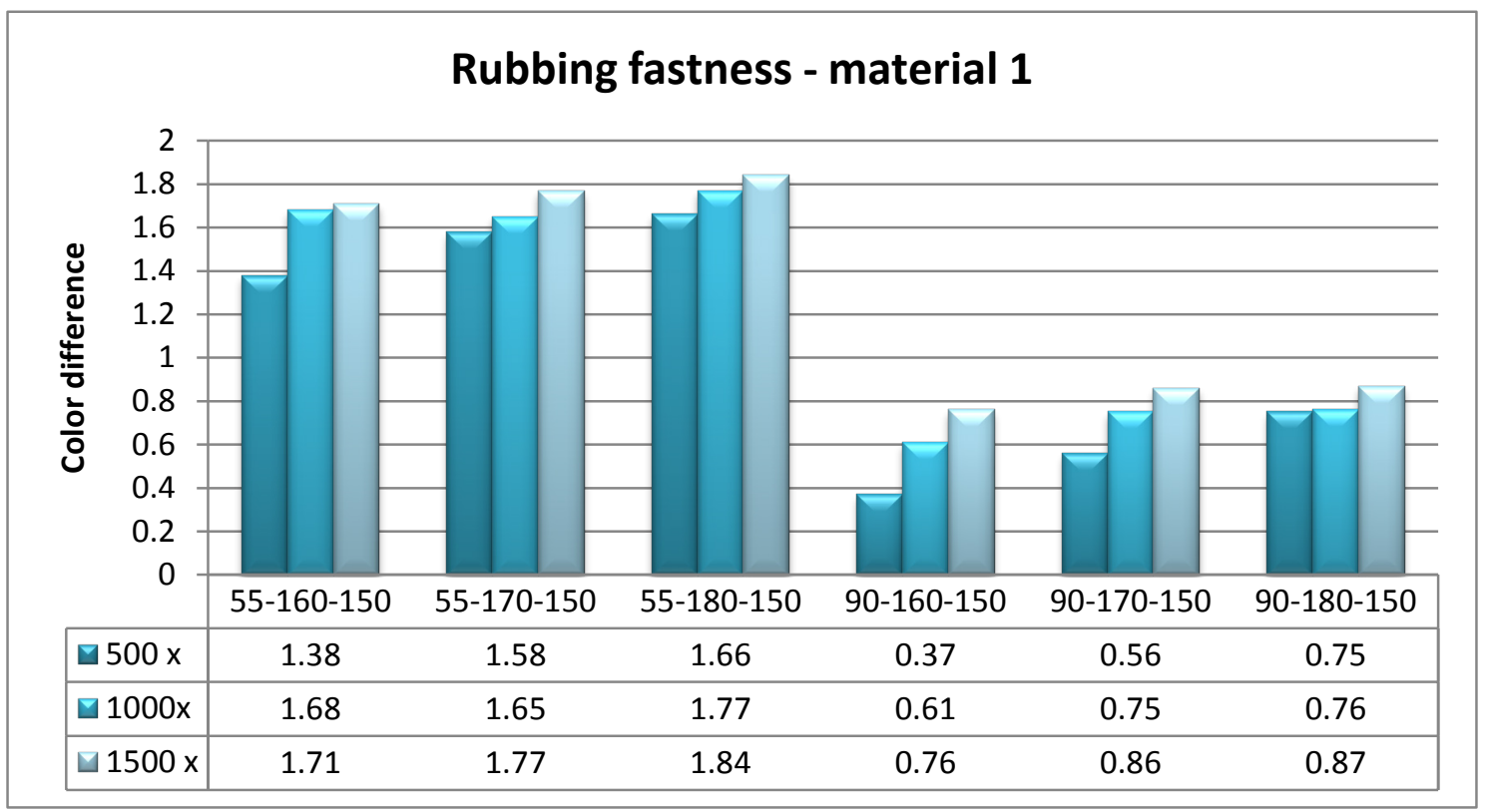

Figure 1: Colour difference between the samples immediately after printing and after rubbing procedure - material 1 (Remark: numbers 55 and 90 represent threads/centimetre, number 160, 170 and 180 represent temperature in ${ }^{\circ} \mathrm{C}$; number 150 represent fabric weight for material 1) 
From the given analysis, it can be seen that a change in colour has occurred with the increase in the rubbing repetition. Likewise, the rise in temperature is increasing in colour difference value. Results do not exceed the value of 2 , which means that difference can be noticed only by "trained" eye. This means that although the device detects changes, the average human see samples as identical. The biggest change in $\Delta \mathrm{E}$ value can be observed in the case where the temperature is highest and number of threads per centimetre is 55. The colour difference value in this example is 1,84 .

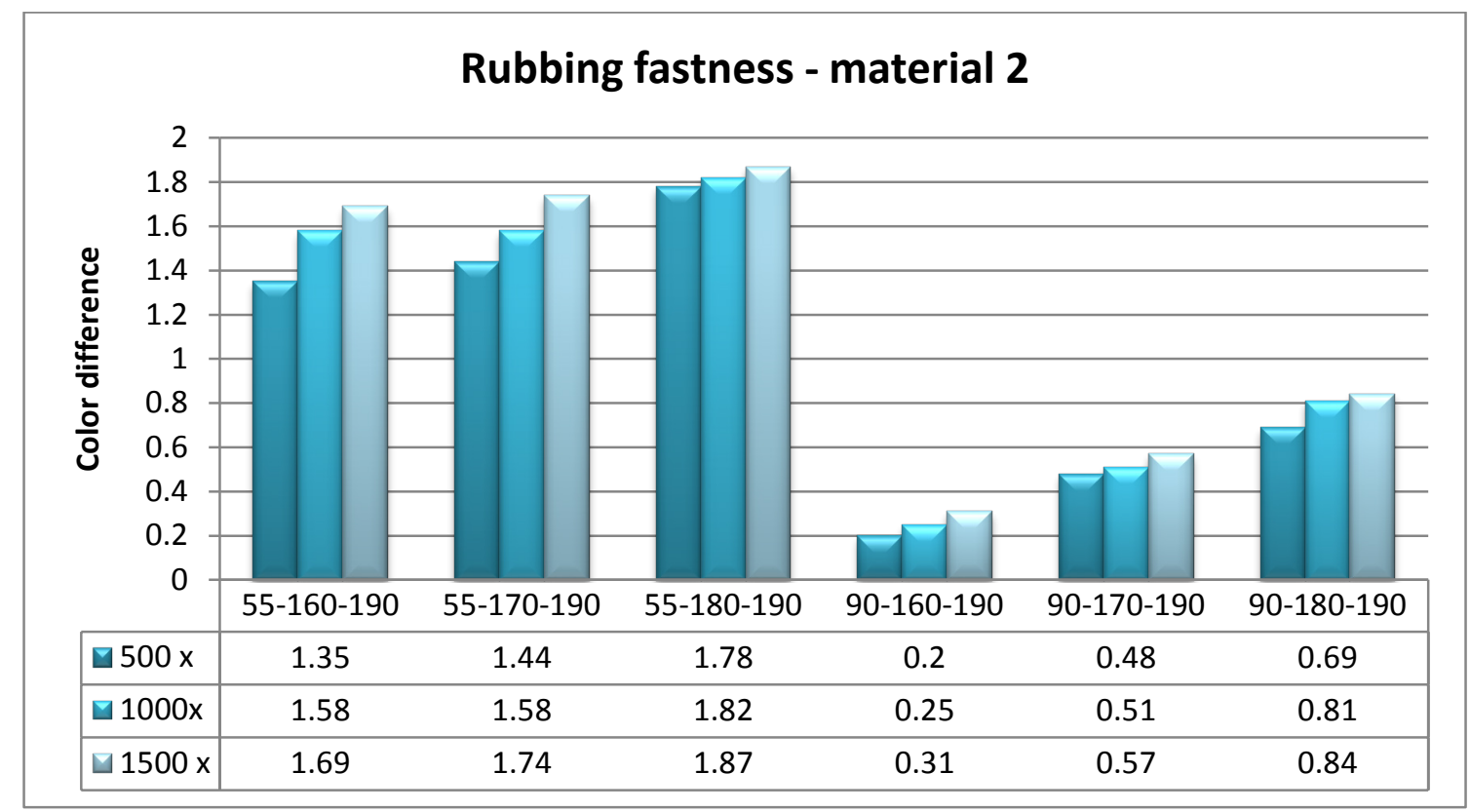

Figure 2: Colour difference between the samples immediately after printing and after rubbing procedure - material 2 (Remark: numbers 55 and 90 represent threads/centimetre, number 160, 170 and 180 represent temperature in ${ }^{\circ} \mathrm{C}$; number 190 represent fabric weight for material 2)

By compering these materials, it has been established that the material 2 provides more permanent proof. This result is consequence of the higher fabric weight. Higher fabric weight can cause the colour to seep deeper into the structure, leaving a smaller layer of paint on the surface that can be damaged. Another characteristic that affects the quality of the print is thread count of screen printing mesh. Prints created with greater thread count screen shows better rubbing fastness as a result of lower amount of ink deposited. The thinner the colour layer is, the less pigments can be removed, resulting in a lower value of colour difference between samples before and after rubbing mechanism. Likewise, lower temperature when transferring paper to material results in better durability of colour.

Figures 3, 4, 5 and 6 show scanned samples using a Canon CanoScan 5600F scanner after rubbing procedure. First samples shown in each group are original samples scanner right after printing process. 
a)
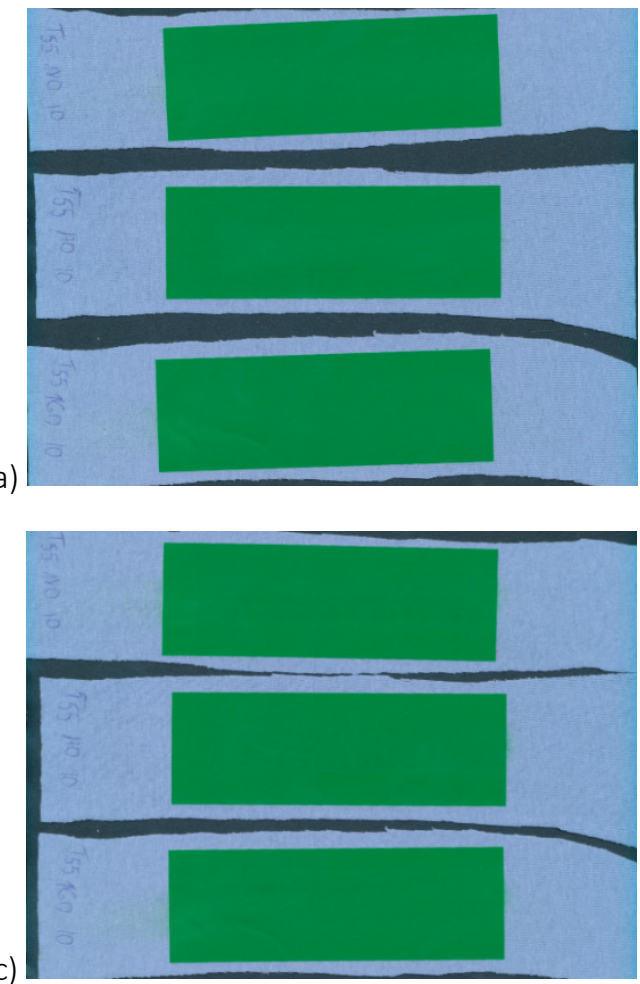

b)

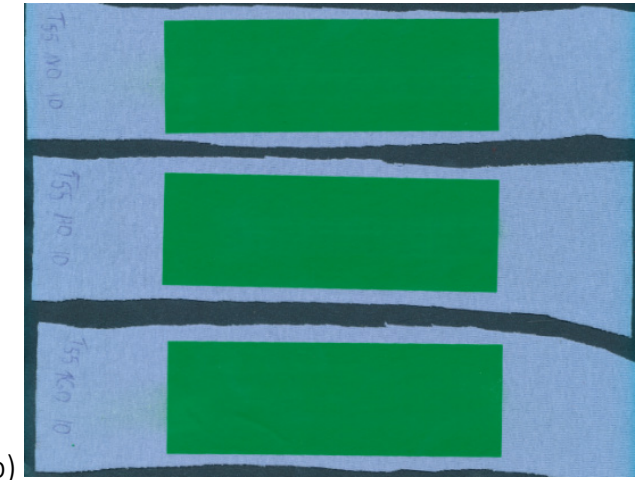

d)

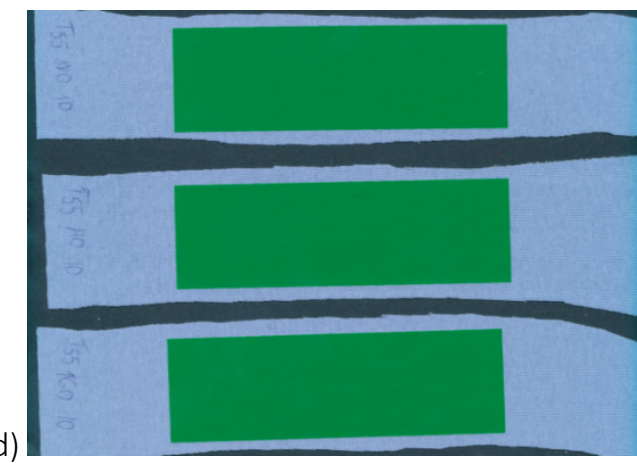

Figure 3 (part 2): Scanned samples (55 thread/cm, 160, 170 and $180^{\circ} \mathrm{C}$ ) after printing process and rubbing treatment - material 1: a) after printing process, b) after $500 \times$ rubbing repetitions, c) after 1000 x rubbing repetitions, d) after 1500 x rubbing repetitions

a)

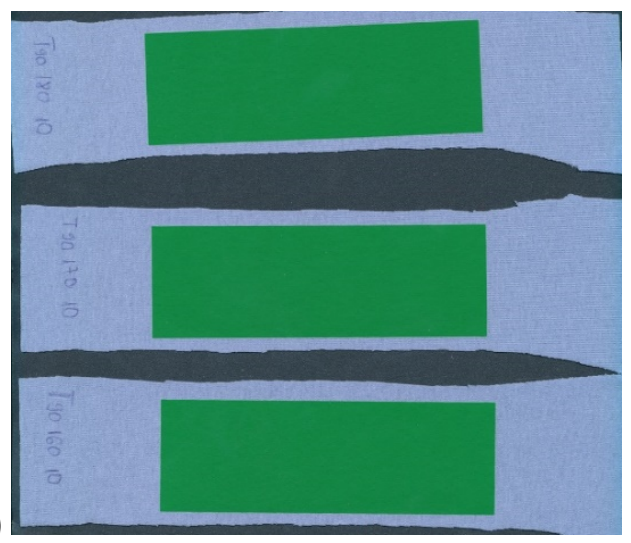

c)

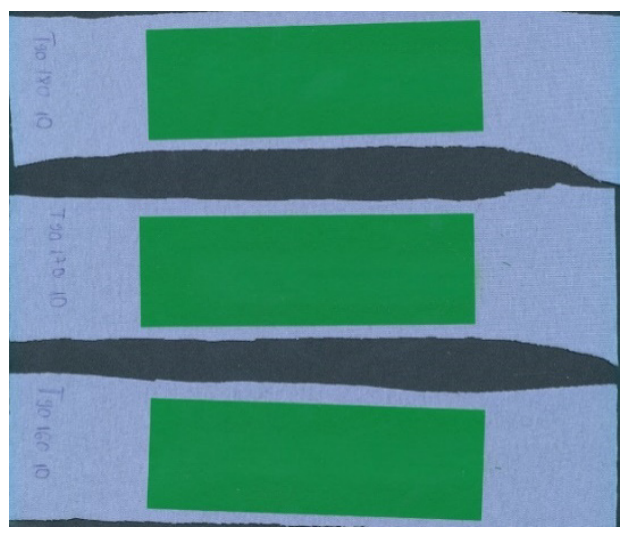

b)

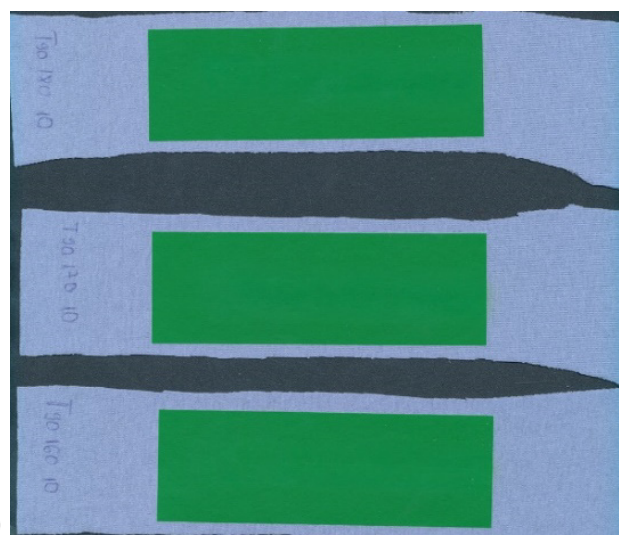

d)

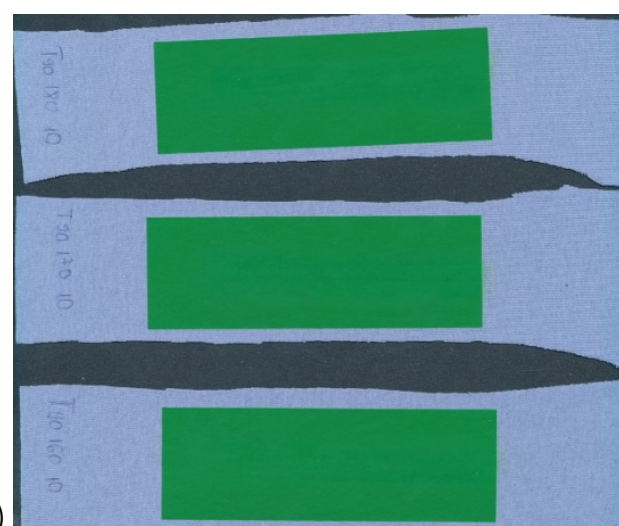

Figure 4: Scanned samples $\left(90\right.$ thread/cm, 160,170 and $\left.180^{\circ} \mathrm{C}\right)$ after printing process and rubbing treatment material 1: a) after printing process, b) after $500 \times$ rubbing repetitions, c) after 1000 x rubbing repetitions, d) after $1500 \times$ rubbing repetitions 

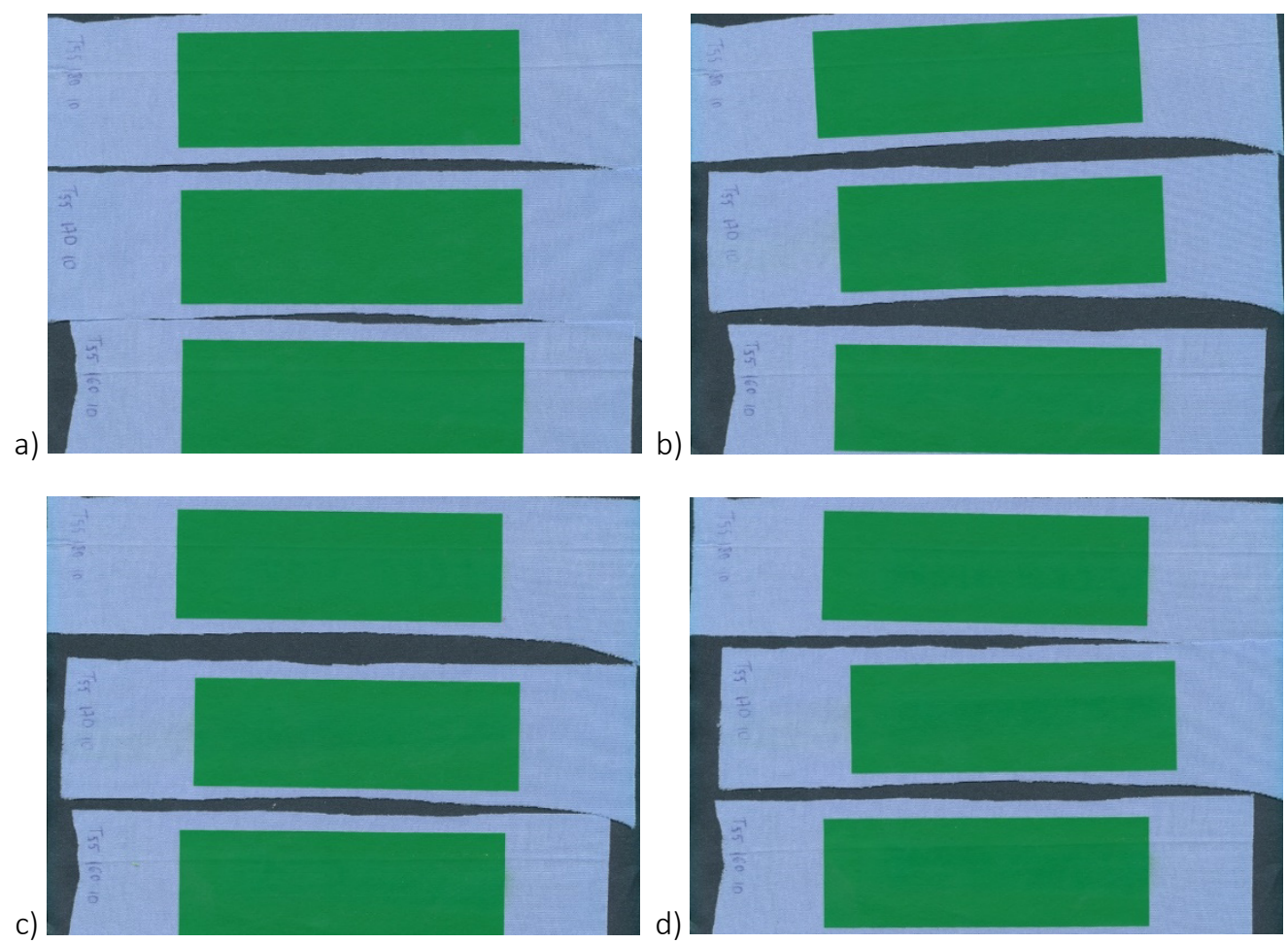

Figure 5: Scanned samples $\left(55\right.$ thread/cm, 160, 170 and $\left.180^{\circ} \mathrm{C}\right)$ after printing process and rubbing treatmentmaterial 2: a) after printing process, b) after $500 \times$ rubbing repetitions, c) after $1000 \times$ rubbing repetitions, d) after $1500 \times$ rubbing repetitions

a)

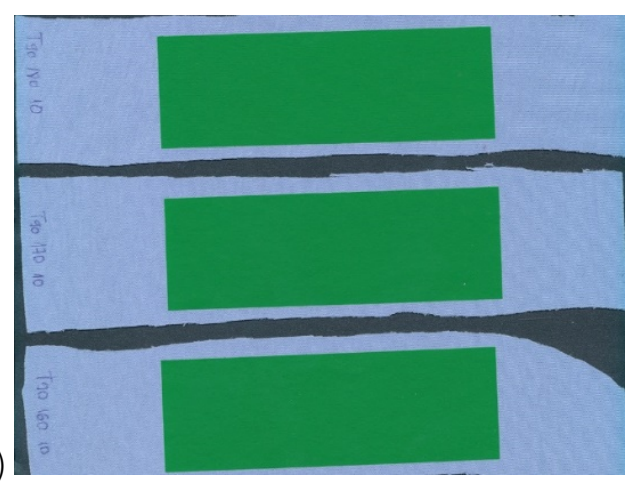

c)

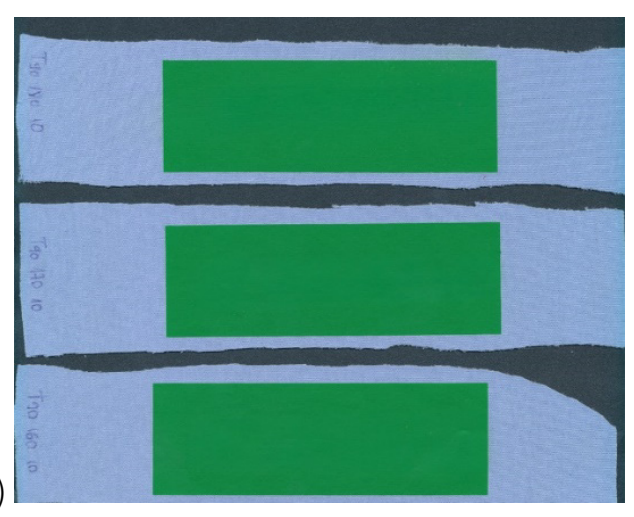

b)

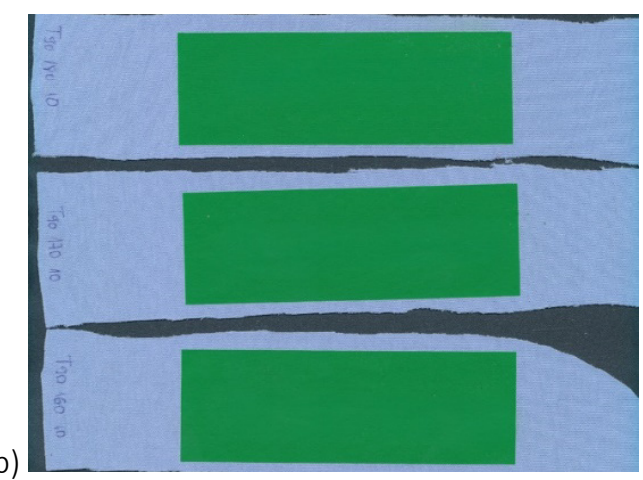

d)

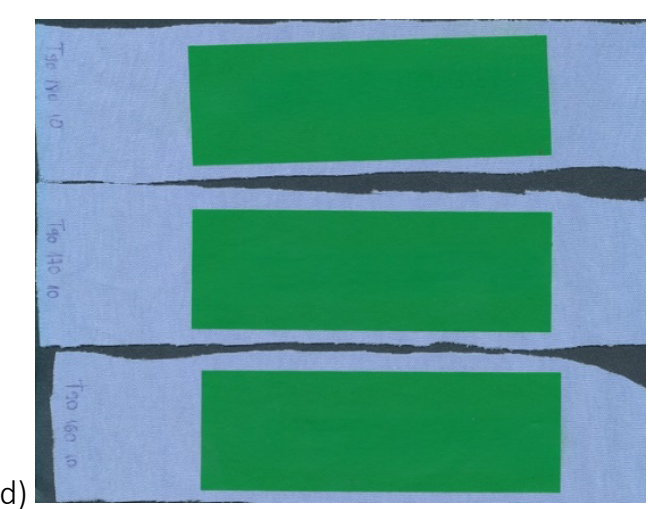

Figure 6: Scanned samples $\left(90\right.$ thread/cm, 160, 170 and $\left.180^{\circ} \mathrm{C}\right)$ after printing process and rubbing treatmentmaterial 2: a) after printing process, b) after $500 \times$ rubbing repetitions,

c) after 1000 x rubbing repetitions, d) after $1500 \times$ rubbing repetitions 
Although measurements were made on two different materials, as well as different screen thread, it can be concluded that there are no visible changes in samples. If the rubbing continues, the colour difference will reach the level at which the eye will notice the difference. However, this study shows that the balance of various factors such as material properties, screen thread count and temperature in transfer process can sustain the quality of print.

\section{CONCLUSIONS}

Transfer printing is highly accepted technique on the market. Some of the advantages are economical aspect such as low cost transfer paper, inexpensive equipment and also fast response to sales demand. This analysis was performed to examine the stability of the prints produced by transfer screen printing in case of rubbing. Although the results showed that there was a difference between the samples immediately after printing and after rubbing procedure, the quality of the prints remained constant. Colour difference values will be higher but those values will not be high enough to cause significant visible damage.

\section{ACKNOWLEDGMENTS}

This work was supported by the Serbian Ministry of Science and Technological Development, Grant No.:35027 "The development of software model for improvement of knowledge and production in graphic arts industry".

\section{REFERENCES}

[1] Cahill, V.: "Digital printing of textiles", 1st ed, (Woodhead Publishing Limited, Abington England, 2006).

[2] Kašiković N., Stančić M., Spiridonov I., Novaković D., Milošević R., Grujić D., Ružičić B.: "The effect of washing temperature and number of washing cycles on the quality of screen printed textiles materials", Bulgarian Chemical Communications 49, 130-139, 2017.

[3] Novaković D., Karlović I., Pavlović Ž., Pešterac Č.: "Reprodukciona tehnika- priručnik za vežbe", (Fakultet tehničkih nauka, Novi Sad, 2008).

[4] Rattee I. D.: "Transfer printing in textile printing", In: Miles, L.W.C. (ed.) Textile Printing, 2003.

[5] Tippet, B. G.:" The evolution and progression of digital textile printing", URL http://brookstippett.com/docs/Print2002-BGT.pdf (last request: 2018-5-22).

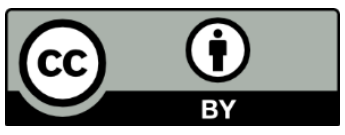

(C) 2018 Authors. Published by the University of Novi Sad, Faculty of Technical Sciences, Department of Graphic Engineering and Design. This article is an open access article distributed under the terms and conditions of the Creative Commons Attribution license 3.0 Serbia (http://creativecommons.org/licenses/by/3.0/rs/). 